\title{
EFEITO DO GRAU DE MOAGEM, DO TIPO DE FRASCO E DO VOLUME VAZIO SOBRE A VARIABILIDADE ANALÍTICA DO FÓSFORO EXTRAÍDO PELOS MÉTODOS MEHLICH-1 E MEHLICH-3 ${ }^{(1)}$
}

\author{
Lenio da Silva Santos ${ }^{(2)}$, Leandro Souza da Silva ${ }^{(3)}$, Alberto Cargnelutti Filho $^{(4)} \&$ \\ Gustavo Griebeler ${ }^{(5)}$
}

\begin{abstract}
RESUMO
Na execução de uma análise química de solo são empregados diversos procedimentos que, mesmo seguindo-se o protocolo preconizado pelo método de análise utilizado, estão sujeitos a variações nos resultados analíticos, causadas por manipulação das amostras ou dos materiais utilizados. Objetivou-se neste estudo avaliar a influência do grau de moagem da amostra, do tipo de frasco e do volume vazio no frasco na execução dos métodos Mehlich-1 e Mehlich-3 para determinar o P no solo. Para tanto, foram conduzidos três experimentos. No primeiro experimento, as amostras de solo foram moídas e tamisadas em peneiras com aberturas de 2,000; 1,$700 ; 0,850 ; 0,600 ;$ e $0,300 \mathrm{~mm}$. No segundo, foram utilizados dois modelos de frasco (erlenmeyer e snap-cap), ambos com volume de $50 \mathrm{~mL}$. Já no terceiro, foi alterado o volume vazio no frasco, mantendo-se a relação solo:solução extratora utilizando-se as quantidades dentro do frasco de $1: 10 ; 1,5: 15 ; 2,5: 25 ; 3: 30$; e 4:40 $\mathbf{c m}^{3} \mathbf{c m}^{-3}$. O grau de moagem das amostras não influenciou a capacidade de extração do Mehlich-1; entretanto, a capacidade extrativa do Mehlich-3 foi influenciada, principalmente em solos argilosos. Tanto para o Mehlich-1 quanto para o Mehlich-3, os teores de $\mathbf{P}$ extraído foram significativamente mais elevados com o uso de frasco tipo snap-cap em relação ao erlenmeyer. $O$ volume vazio no frasco alterou os teores de $P$ extraído para o Mehlich-1 e Mehlich-3 em 100 e 64 \% das amostras, respectivamente. Deve-se padronizar a intensidade da moagem das amostras de solo para extração do $P$ pela solução de Mehlich-3. Um modelo único de frasco deve ser adotado pelos laboratórios de rotina para análise do $\mathrm{P}$,
\end{abstract}

(1) Parte da Dissertação de Mestrado do primeiro autor, apresentada à Universidade Federal de Santa Maria - UFSM. Recebido para publicação em 7 de outubro de 2013 e aprovado em 2 de junho de 2014 .

(2) Mestrando do Programa de Pós-Graduação em Ciência do Solo, UFSM. Av. Roraima, 1000, Camobi. CEP 97105-900 Santa Maria (RS). E-mail: lenio2501@hotmail.com

(3) Professor Associado, Departamento de Solos, UfSM. Bolsista de Produtividade em Pesquisa do CNPq. E-mail: leandrosolos@ufsm.br

(4) Professor Associado, Departamento de Fitotecnia, UFSM. Bolsista de Produtividade em Pesquisa do CNPq. E-mail: cargnelutti@pq.cnpq.br

(5) Doutorando do Programa de Pós-Graduação em Ciência do Solo, UFSM. E-mail: gustavogriebeler@hotmail.com 
independentemente do método de extração, mantendo-se sempre constante no frasco o volume da amostra $\left(\mathrm{cm}^{3}\right)$ para o volume de solução extratora $\left(\mathrm{cm}^{3}\right)$.

Termos de indexação: análise de solo, método de extração, procedimentos analíticos.

\title{
SUMMARY: ANALYTICAL VARIABILITY OF PHOSPHORUS EXTRACTED BY MEHLICH-1 AND MEHLICH-3 CAUSED BY SOIL PARTICLE SIZE, CONTAINER TYPE AND CONTAINER VOID VOLUME
}

\begin{abstract}
Even following the protocol recommended by the method of analysis, variations in analytical results can be caused by the way samples, or the kind of materials used in the analysis, are handled. The objective of this study was to evaluate the influence of soil particle size, the type of extraction container, and the container void volume on soil P content extracted by Mehlich-1 and Mehlich-3. In the first experiment, soil samples were ground to pass through sieves with 2.000, 1.700, 0.850, 0.600, and $0.300 \mathrm{~mm}$ diameter openings. In the second experiment two $50 \mathrm{~mL}$ container types (Erlenmeyer and "snap-cap") were used. In the third experiment the void volume of the container was changed while maintaining the soil:extraction solution ratio within the container at 1:10, 1.5:15, 2.5:25; $3: 30$ and 4:40 $\mathrm{cm}^{3} \mathrm{~cm}^{-3}$, respectively. Soil particle size of the samples did not affect the extraction capacity of Mehlich-1; however, the extraction capacity of Mehlich-3 was affected, especially in clay soils. Extracted P was significantly higher with "snap-cap" type containers than Erlenmeyer containers for both methods. The void volume of the container affected the content of Pextracted by Mehlich-1 and Mehlich-3 in 100 and $64 \%$ of the samples, respectively. We recommend standardizing soil particle size for $P$ extraction by the Mehlich-3 solution. A single container type should be adopted by laboratories for P analysis, regardless of the extraction method, always maintaining the sample volume $\left(\mathrm{cm}^{3}\right)$ to extraction solution volume $\left(\mathrm{cm}^{3}\right)$ contained in the bottle constant.
\end{abstract}

Index terms: soil analysis, phosphorus extraction method, analytical procedures.

\section{INTRODUÇÃO}

O método empregado pelos laboratórios de análises de solo dos Estados do Rio Grande do Sul e Santa Catarina para extrair o $\mathrm{P}$ considerado disponível às plantas é o Mehlich-1 (M1), adaptado por Tedesco et al. (1995). Esse método possui como vantagens ser de fácil execução, baixo custo e apresentar extratos límpidos, condição essa essencial à determinação por espectrometria de absorção molecular UV/Visível (colorimetria). Porém, esse método tem apresentado elevado coeficiente de variação nos dados na determinação do $\mathrm{P}$ pelo programa de Controle de Qualidade da Rede Oficial dos Laboratórios de Análises de Solos dos Estados do Rio Grande do Sul e de Santa Catarina (ROLAS-RS/SC, 2012). Tal comportamento pode estar relacionado com variações no procedimento analítico empregado pelos laboratórios, mesmo que o protocolo geral preconizado pelo método seja seguido.

Existem outros métodos de avaliação do $\mathrm{P}$ no solo disponível às plantas, com princípios de extração variados (Silva \& Raij, 1999). O uso de extratores multielementares, como o Mehlich-3 (M3), em conjunto com a técnica da espectrometria de emissão ótica por plasma induzido (ICP-OES), possibilitaria discriminar no mesmo extrato, além do $\mathrm{P}$, outros elementos (Sikora et al., 2005), o que simplificaria os procedimentos analíticos e aumentaria a eficiência dos laboratórios de análises de solo (Bortolon \& Gianello,
2010). Entretanto, outros métodos de análise de $\mathrm{P}$ também podem estar sujeitos a certa variabilidade nos resultados decorrentes de variações nos procedimentos analíticos, mas com magnitudes de acordo com o princípio de extração/determinação de cada método e a natureza da variação causada no procedimento. A moagem faz-se necessária para tomada de alíquotas por volume (cachimbagem) na análise de solo, além de aumentar a homogeneidade da amostra (Raij et al., 1987). Entretanto, a intensidade dessa moagem pode influenciar o valor extraído de P. À medida que o tamanho das partículas diminui as soluções extratoras podem acessar/extrair diferentes formas de P, que, em condições naturais, não estariam disponíveis para as plantas (Makarov et al., 2004; Wang et al., 2006; Rolim et al., 2008). Outras variáveis importantes e, muitas vezes negligenciadas nos laboratório de análises de solo, são o tamanho/tipo do frasco para a extração e a quantidade de amostra e solução utilizadas nesses, visto que, à medida que se modifica o frasco ou se altera a quantidade de solo e de solução, mesmo que a relação solo:solução preconizada pelo método (1:10) seja mantida, ocorre o aumento ou diminuição do volume vazio no frasco e, consequentemente, do turbilhonamento no momento da agitação. Essa modificação provoca alterações na velocidade de colisões das moléculas, e a taxa da reação de extração de $\mathrm{P}$ pode vir a ser influenciada por esses fatores (Vollhardt \& Schore, 2004). Assim, em razão de os 
métodos M1 e M3 extraírem o P presente no solo por meio de uma reação química (Brasil \& Muraoka, 1997; Bortolon, 2005; Rheinheimer et al., 2008), pequenas modificações nas etapas do protocolo de execução do método podem interferir na qualidade dos resultados analíticos, constituindo num problema, pois resultados discrepantes podem comprometer a interpretação dos resultados.

Dessa forma, o objetivo deste estudo foi avaliar os efeitos do grau de moagem de amostras de solo, do formato do frasco de agitação e do volume vazio no frasco de agitação sobre a variabilidade do $\mathrm{P}$ pelos extratores M1 e M3.

\section{MATERIAL E MÉTODOS}

O estudo foi conduzido no Laboratório de Química e Fertilidade do Solo da Universidade Federal de Santa Maria e no Laboratório de Análises de Solos da Universidade Regional Integrada do Alto Uruguai e das Missões - URI, Campus de Frederico Westphalen, utilizando 11 amostras de solo das classes Latossolo, Neossolo, Argissolo, Cambissolo, Planossolo, coletadas na camada superficial $(0-20 \mathrm{~cm})$ nas regiões geomorfológicas do Planalto e da Depressão Central do Estado do Rio Grande do Sul, as quais apresentam ampla variação dos atributos físicos, químicos e mineralógicos (Quadros 1 e 2). Foram selecionadas amostras com diferentes teores de argila por causa do efeito dessa sobre a capacidade de extração da solução $\mathrm{M} 1$ e com diferentes níveis de disponibilidade de $\mathrm{P}$ (CQFSRS/SC, 2004).

As amostras de solos foram secas em estufa com circulação de ar forçada durante $48 \mathrm{~h}$ à temperatura de $40-45^{\circ} \mathrm{C}$. Após a secagem, foi realizada a moagem das amostras em moinho de martelos e essas tamisadas em peneira de malha de $2,000 \mathrm{~mm}$, com posterior armazenamento em sacos plásticos. Foram avaliados o método M1 proposto por Mehlich (1953), adaptado por Tedesco et al. (1995), e o método M3, com adaptações descritas por Schlindwein (2003). Para a extração do M3, utilizou-se frasco erlenmeyer de 50 $\mathrm{mL}$ idêntico ao do M1 e agitação em mesa pendular horizontal. Foi considerado como referência o $\mathrm{P}$ extraído pela solução de $\mathrm{M} 1\left(\mathrm{HCl} 0,05 \mathrm{~mol} \mathrm{~L}^{-1}+\mathrm{H}_{2} \mathrm{SO}_{4}\right.$ $0,0125 \mathrm{~mol} \mathrm{~L}^{-1}$ ), quando utilizados $3 \mathrm{~cm}^{3}$ de solo e $30 \mathrm{~mL}$ de solução extratora (relação 1:10), com agitação por $5 \mathrm{~min}$. Usou-se para agitar a mistura uma mesa agitadora horizontal pendular de bancada com plataforma de movimento longitudinal com amplitude de $50 \mathrm{~mm}$ e dimensões de $700 \times 540 \times 370 \mathrm{~mm}(\mathrm{~L} \times$ $\mathrm{P} \times \mathrm{A}$ ) a 120 oscilações por min e decantação por $16 \mathrm{~h}$, de acordo com método descrito por Tedesco et al. (1995). O procedimento de referência de extração do $\mathrm{P}$ com a solução de $\mathrm{M} 3\left(\mathrm{CH}_{3} \mathrm{COOH} 0,2 \mathrm{~mol} \mathrm{~L}^{-1}+\right.$ $\mathrm{NH}_{4} \mathrm{NO}_{3} 0,25 \mathrm{~mol} \mathrm{~L}^{-1}+\mathrm{NH}_{4} \mathrm{~F} 0,015 \mathrm{~mol} \mathrm{~L}^{-1}+\mathrm{HNO}_{3}$ 0,013 mol L-1 + EDTA 0,001 $\mathrm{mol} \mathrm{L}^{-1}$ ) também teve relação de solo:solução de 1:10, com agitação em mesa pendular horizontal a 120 oscilações por min. A etapa de filtragem dos extratos foi substituída por decantação por $16 \mathrm{~h}$, conforme Bortolon \& Gianello (2008). O teor de P nos extratos das amostras de ambos os métodos foi definido por espectrofotometria de absorção molecular UV/Vis (colorimetria) num colorímetro Genesys 20.

$\mathrm{O}$ estudo foi realizado em três experimentos, em que foram simuladas variações nas deferentes etapas (moagem, tipo de frasco, volume vazio no frasco) do protocolo de análise do $\mathrm{P}$ estabelecidos anteriormente, com a finalidade de identificar a magnitude dessas alterações sobre os teores de P extraído.

\section{Moagem das amostras}

A partir das amostras moídas e tamisadas em peneira com malha de $2,000 \mathrm{~mm}$, foram retiradas subamostras, as quais foram moídas em almofariz até passarem totalmente em peneiras $\mathrm{ABNT}$ com abertura 1,$700 ; 0,850 ; 0,600$; e $0,300 \mathrm{~mm}$. Toda a subamostra retirada foi forçada a passar na respectiva malha, a fim de minimizar uma possível segregação

Quadro 1. Identificação das amostras de solos

\begin{tabular}{|c|c|c|c|c|c|}
\hline Legenda & $\begin{array}{l}\text { Classe } \\
\text { de solo }\end{array}$ & $\begin{array}{c}\text { Local de coleta } \\
\text { (município) }\end{array}$ & $\begin{array}{l}\text { Uso do } \\
\text { solo }\end{array}$ & $\begin{array}{c}\text { Classe } \\
\text { textural }\end{array}$ & $\begin{array}{c}\text { Nível de } \\
\text { suficiência de } P\end{array}$ \\
\hline $\mathrm{LCm} \mathrm{1/A}$ & Latossolo & Condor & mato & $1(>60 \%)$ & Alto (A) \\
\hline $\mathrm{LCl} 1 / \mathrm{A}$ & Latossolo & Condor & lavoura & $1(>60 \%)$ & Alto (A) \\
\hline LFWc 1/B & Latossolo & Frederico Westphalen & campo & $1(>60 \%)$ & Baixo (B) \\
\hline $\mathrm{LBVm} \mathrm{1/B}$ & Latossolo & B. V. das Missões & mato & $1(>60 \%)$ & Baixo (B) \\
\hline NFWm 2/MB & Neossolo & Frederico Westphalen & mato & $2(41 \leq 60 \%)$ & Muito baixo (MB) \\
\hline NFWc 2/MB & Neossolo & Frederico Westphalen & campo & $2(41 \leq 60 \%)$ & Muito baixo (MB) \\
\hline PSMl 2/A & Planossolo & Santa Maria & lavoura & $2(41 \leq 60 \%)$ & Alto (A) \\
\hline PSMl 3/A & Planossolo & Santa Maria & lavoura & $3(21 \leq 40 \%)$ & Alto (A) \\
\hline PSMl 3/M & Planossolo & Santa Maria & lavoura & $3(21 \leq 40 \%)$ & Médio (M) \\
\hline AIl 3/M & Argissolo & Itaara & lavoura & $3(21 \leq 40 \%)$ & Médio (M) \\
\hline $\mathrm{CBGm} \mathrm{3/M}$ & Cambissolo & Bento Gonçalves & mato & $3(21 \leq 40 \%)$ & Médio (M) \\
\hline
\end{tabular}


Quadro 2. Caracterização física e química das amostras de solos

\begin{tabular}{|c|c|c|c|c|c|c|c|c|c|c|c|}
\hline Atributo & $\begin{array}{c}\mathrm{LCm} \\
1 / \mathrm{A}\end{array}$ & $\begin{array}{c}\text { LC1 } \\
\text { 1/A }\end{array}$ & $\begin{array}{c}\text { LFWc } \\
1 / B\end{array}$ & $\begin{array}{c}\text { LBVm } \\
1 / B\end{array}$ & $\begin{array}{c}\text { PSM1 } \\
\text { 2/A }\end{array}$ & $\begin{array}{c}\text { NFWm } \\
2 / M B\end{array}$ & $\begin{array}{c}\text { NFWc } \\
2 / \mathrm{MB}\end{array}$ & $\begin{array}{c}\text { PSM1 } \\
\text { 3/A }\end{array}$ & $\begin{array}{l}\text { AI1 } \\
\text { 3/M }\end{array}$ & $\begin{array}{c}\text { CBGm } \\
3 / M\end{array}$ & $\begin{array}{c}\text { PSM1 } \\
\text { 3/M }\end{array}$ \\
\hline Areia $\left(\mathrm{g} \mathrm{kg}^{-1}\right)^{(1)}$ & 213 & 244 & 96 & 75 & 191 & 110 & 120 & 316 & 445 & 303 & 461 \\
\hline Silte $\left(\mathrm{g} \mathrm{kg}^{-1}\right)^{(1)}$ & 102 & 171 & 120 & 140 & 384 & 305 & 315 & 339 & 250 & 332 & 274 \\
\hline Argila $\left(\mathrm{g} \mathrm{kg}^{-1}\right)^{(1)}$ & 685 & 585 & 785 & 785 & 425 & 585 & 565 & 345 & 305 & 365 & 265 \\
\hline $\mathrm{pH}\left(\mathrm{H}_{2} \mathrm{O}\right) \quad 1: 1$ & 4,7 & 5,5 & 4,8 & 4,6 & 5,0 & 5,3 & 5,4 & 4,8 & 5,1 & 5,7 & 4,8 \\
\hline Índice $\mathrm{SMP}^{(2)}$ & 5,2 & 6,0 & 5,4 & 5,3 & 5,3 & 5,9 & 5,7 & 5,3 & 5,6 & 6,5 & 5,0 \\
\hline $\mathrm{P}\left(\mathrm{mg} \mathrm{dm} \mathrm{m}^{-3}\right)^{(3)}$ & 9,1 & 8,3 & 2,2 & 2,6 & 9,8 & 2,5 & 1,7 & 20,4 & 9,3 & 11,1 & 8,8 \\
\hline $\mathrm{K}\left(\mathrm{mg} \mathrm{dm} \mathrm{m}^{-3}\right)^{(3)}$ & 77 & 159 & 14 & 67 & 342 & 321 & 59 & 141 & 203 & 176 & 117 \\
\hline $\mathrm{P}\left(\mathrm{mg} \mathrm{dm}^{-3}\right)^{(4)}$ & 10,2 & 9,5 & 2,2 & 3,1 & 13,3 & 2,5 & 1,8 & 24,1 & 12,7 & 17,8 & 12,4 \\
\hline $\operatorname{MO}(\%)\left(\mathrm{m} \mathrm{m}^{-1}\right)^{(5)}$ & 3,3 & 3,0 & 2,1 & 5,0 & 4,5 & 5,6 & 3,7 & 3,2 & 3,2 & 3,9 & 2,9 \\
\hline $\mathrm{Al}^{3+}\left(\mathrm{cmol}_{\mathrm{c}} \mathrm{dm}^{-3}\right)^{(6)}$ & 4,2 & 0,2 & 3,8 & 2,8 & 1,8 & 0,2 & 0,3 & 4,1 & 0,8 & 0,1 & 7,2 \\
\hline $\mathrm{Ca}^{2+}\left(\mathrm{cmol}_{\mathrm{c}} \mathrm{dm}^{-3}\right)^{(6)}$ & 0,6 & 2,5 & 0,1 & 2,3 & 4,6 & 6,6 & 4,6 & 2,1 & 2,9 & 5,6 & 0,1 \\
\hline $\mathrm{Mg}^{2+}\left(\mathrm{cmol}_{\mathrm{c}} \mathrm{dm}^{-3}\right)^{(6)}$ & 0,3 & 1,6 & 0,2 & 0,6 & 2,9 & 1,4 & 0,5 & 0,7 & 0,7 & 0,9 & 0,1 \\
\hline $\left.\mathrm{Mn}^{2+}(\mathrm{mg} \mathrm{dm})^{-3}\right)^{(3)}$ & 64 & 23 & 94 & 226 & 69 & 132 & 103 & 50 & 114 & 5 & 22 \\
\hline $\mathrm{Cu}^{2+}\left(\mathrm{mg} \mathrm{dm}^{-3}\right)^{(3)}$ & 7,7 & 9,4 & 18,5 & 6,2 & 1,6 & 17,7 & 27 & 1,6 & 42,6 & 0,9 & 1,2 \\
\hline $\mathrm{Zn}^{2+}\left(\mathrm{mg} \mathrm{dm}^{-3}\right)^{(3)}$ & 1,9 & 2,6 & 3,4 & 4,6 & 3,2 & 11,2 & 12,2 & 2,0 & 7,2 & 9,8 & 1,1 \\
\hline $\mathrm{Fe}_{\mathrm{o}}\left(\mathrm{mg} \mathrm{kg}^{-1}\right)^{(7)}$ & 76,0 & 46,0 & 68,0 & 38,0 & 86,0 & 70,0 & 60,0 & 86,0 & 38,0 & 70,0 & 84,0 \\
\hline $\mathrm{Fe}_{\mathrm{d}}\left(\mathrm{g} \mathrm{kg}^{-1}\right)^{(8)}$ & 19,7 & 26,5 & 40,5 & 33,4 & 4,4 & 33,9 & 35,9 & 1,7 & 10,0 & 14,4 & 3,4 \\
\hline
\end{tabular}

(1) Método do densímetro de Bouyoucos; (2) Solução tampão a pH 7,5; (3) Método Mehlich-1; (4) Método Mehlich-3, conforme Schlindwein (2003); ${ }^{(5)}$ MO: matéria orgânica, combustão úmida; ${ }^{(6)}$ Cloreto de potássio 1,0 mol L ${ }^{-1}$; (7) Oxalato de amônio 0,2 mol L-1; e ${ }^{(8)}$ Ditionito-bicarbonato-citrato, conforme Mehra \& Jackson (1964).

de partículas e de agregados, os quais fazem parte da amostra original e não são totalmente moídos pelo procedimento de referência. Dessa forma, eliminouse uma possível alteração dos resultados analíticos em razão da mudança na composição das frações areia, argila e silte característica de cada solo.

\section{Tipo de frasco}

$\mathrm{Na}$ avaliação do efeito do tipo de frasco sobre os teores extraídos de P pelos métodos M1 e M3, foram utilizados dois modelos de frasco com volume total de $50 \mathrm{~mL}$. O primeiro foi o frasco erlenmeyer de boca estreita recomendado por Tedesco et al. (1995); e o segundo foi um frasco tipo snap-cap. As dimensões dos frascos são apresentadas na figura 1.

\section{Volume vazio no frasco}

A verificação do efeito do volume vazio no frasco foi realizada promovendo alteração na quantidade da relação volume de solo:solução dentro do frasco; o padrão é 3:30 para as proporções 1:10; 1,5:15; 2,5:25; e $4: 40 \mathrm{~cm}^{3} \mathrm{~cm}^{-3} \mathrm{em}$ frasco erlenmeyer de boca estreita com volume de $50 \mathrm{~mL}$, representando um volume vazio no frasco de 6,$0 ; 17,0 ; 22,5 ; 33,5$; e 39,0 mL.

\section{Análise estatística}

Os experimentos com grau de moagem do solo e volume vazio no frasco foram dispostos em delineamento inteiramente casualizado, num esquema fatorial $2 \times 5 \times 11$ : métodos $\times$ variável $\times$ solos, com quatro repetições. Os resultados foram submetidos à (a)

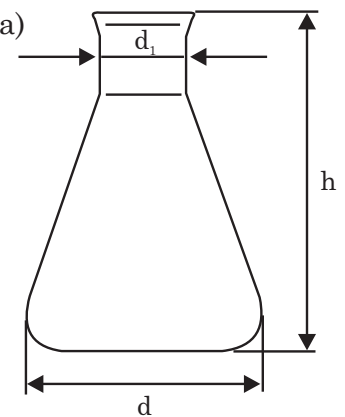

$\mathrm{h}=90,4 \mathrm{~mm} \quad \mathrm{~d}=51,1 \mathrm{~mm} \quad \mathrm{~d}_{1}=19,2 \mathrm{~mm}$ (b)

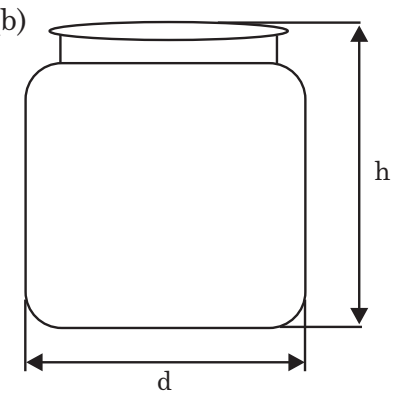

$\mathrm{h}=61,24 \mathrm{~mm} \mathrm{~d}=47,58 \mathrm{~mm}$
Figura 1. Dimensões dos frascos de $50 \mathrm{~mL}$ utilizados no estudo: a) erlenmeyer e b) snap-cap.

análise de variância e, quando os efeitos foram significativos, foram ajustadas equações de regressão para os dados quantitativos. Para a escolha das equações, foram testados os modelos linear, quadrático e cúbico pelo teste $\mathrm{F}$, escolhendo-se aquele com significância maior que $95 \%(p<0,05)$ e, no caso de mais de um modelo ser significativo, optou-se pelo de maior grau. Para os dados qualitativos, quando da significância observada na análise da variância, foram submetidos ao teste de comparação de médias de Tukey, tomando por base os níveis de significância maiores que $95 \%(p<0,05)$. O experimento do tipo de frasco foi disposto em um delineamento inteiramente casualizado num esquema fatorial $2 \times 2 \times 11$ : métodos $\times$ frasco $\times$ solos, com quatro repetições. Foi realizada a análise de variância para fonte de variação, em que, 
para o fator qualitativo, foi aplicado o teste de comparação de médias de Tukey a $5 \%$. Todas as análises estatísticas foram realizadas pelo programa computacional Sistema para Análise de Variância SISVAR (Ferreira, 2008).

\section{RESULTADOS E DISCUSSÃO}

Os três fatores apresentaram interação significativa $(p<0,05)$ nos três experimentos. Porém, o fator solo pode ser desconsiderado da comparação por causa de as amostras utilizadas pertencerem a classes de solos distintas e terem sido, propositalmente, coletadas em locais com diferentes condições de gênese e uso, o que naturalmente determina o teor de $\mathrm{P}$ variado das amostras, sendo, portanto, independente dos métodos de extração e da fonte de variabilidade aplicada nos tratamentos. No quadro 3 e nas figuras 2 e 3 , são apresentados os solos que tiveram efeito significativo.

\section{Efeito do grau da moagem das amostras}

Em relação ao grau de moagem das amostras, houve efeito significativo $(\mathrm{p}<0,05)$ para o $\mathrm{P}$ extraído pelo extrator M1 somente no solo PSMl 3/A, em que o valor do $\mathrm{P}$ foi menor na moagem em malha de $0,600 \mathrm{~mm}$, o que pode ter ocorrido ao acaso já que não há tendência lógica (Figura 2). Rolim et al. (2008), estudando efeito da moagem do solo sobre o teor de $\mathrm{P}$ extraído pelo M1, não observaram efeito em razão da moagem mais intensa das amostras. É provável que o acesso a novas formas expostas de $\mathrm{P}$ com a moagem seja compensado pelo aumento do desgaste do extrator M1, por causa do consumo dos íons hidrogênio $\left(\mathrm{H}^{+}\right)$e sulfato $\left(\mathrm{SO}_{4}{ }^{2-}\right)$ pelas bordas dos grupos funcionais expostos pela ação da moagem e não ocupados pelo $\mathrm{P}$ nos coloides inorgânicos e também pela possível maior readsorção de $\mathrm{P}$ aos coloides durante a extração (Cajuste \& Kussow, 1974; Bahia Filho et al.,1983).

Para o extrator M3, houve efeito significativo do grau de moagem nos solos PSMl 2/A, PSMl 3/A, AIl 3/M, CBGm 3/M e PSMl 3/M, representando $45 \%$ das amostras utilizadas. Essa diferença significativa, provavelmente, deve-se ao fato de o extrator M3 extrair preferencialmente o $\mathrm{P}$ ligado a $\mathrm{Fe}$ e $\mathrm{Al}$ e, em menor proporção, o P ligado a Ca (Rheinheimer et al., 2008) e não basear a extração do $\mathrm{P}$ disponível somente na dissolução ácida, como o M1. Dessa forma, o extrator $\mathrm{M} 3$ acessa formas de $\mathrm{P}$ que na moagem com peneira
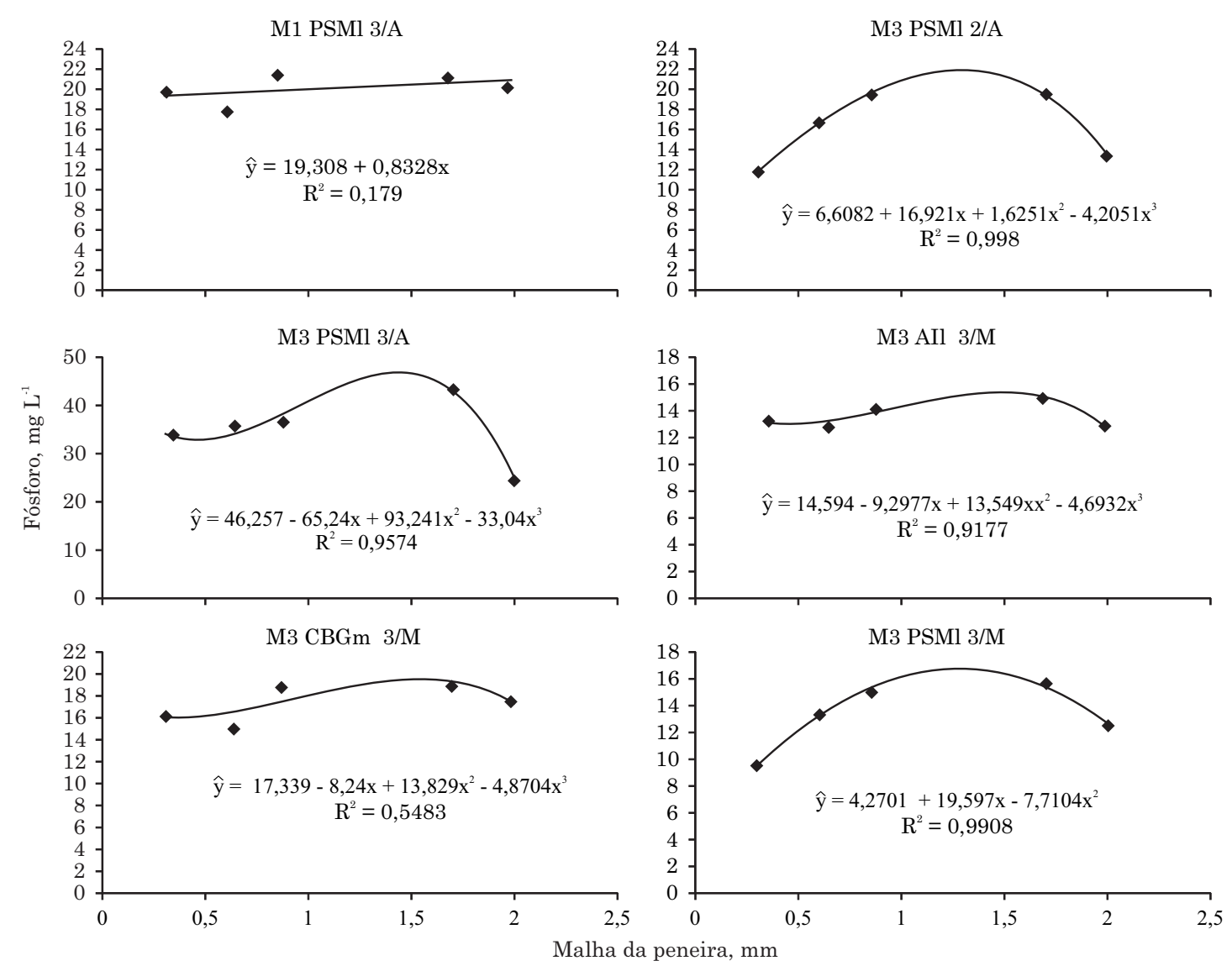

Figura 2. Regressão entre os teores de P extraído pelas soluções Mehlich-1 e Mehlich-3, em diferentes solos e graus de moagem. 

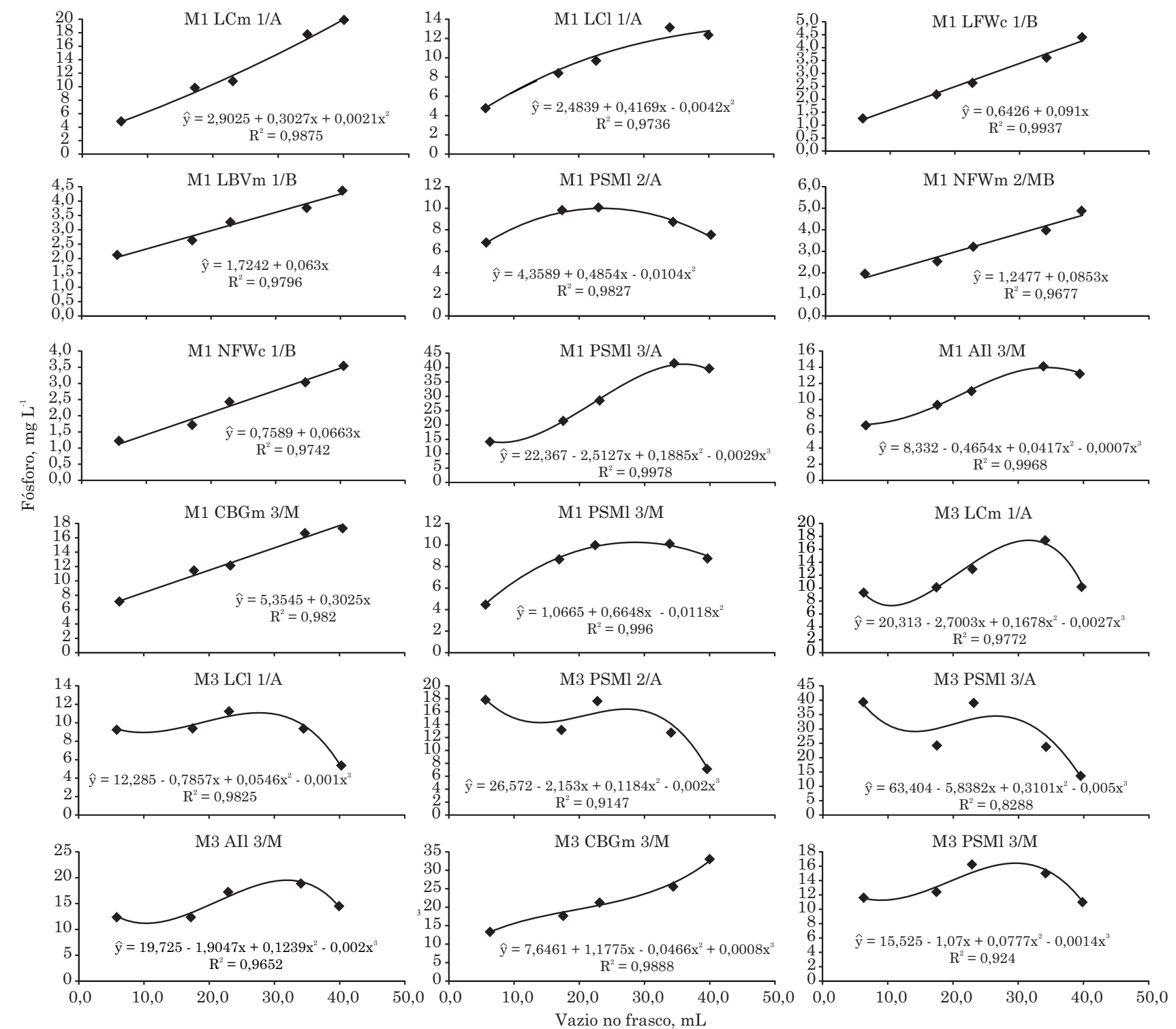

Figura 3. Regressão entre os teores de P extraído pelas soluções Mehlich-1 e Mehlich-3, em diferentes solos e o volume vazio do frasco (relação solo:solução).

de malha 2,000 mm não estariam disponíveis. Contudo, em relação aos solos LFWc 1/B e LBVm 1/B, (argila $>600 \mathrm{~g} \mathrm{dm}^{-3}$ e baixo teor de $\mathrm{P}$ ) e NFWm $2 / \mathrm{MB}$ e NFWc $2 / \mathrm{MB}$ (argila entre 410 e $600 \mathrm{~g} \mathrm{dm}^{-3} \mathrm{e}$ teor de P muito baixo), não houve efeito significativo $(p<0,05)$. Isso, provavelmente, ocorreu pelo rigor do teste estatístico, tendo em vista que em solos muito argilosos e com baixo $\mathrm{P}$ as diferenças são de grandeza menor e acabando por não se identificar a magnitude do efeito da moagem sobre a capacidade do extrator M3. De qualquer forma, os efeitos práticos dessa baixa variabilidade também tendem a ser menos relevantes.

Nos extratos utilizados no M3 em que ocorreu efeito significativo do grau da moagem, houve tendência de comportamento polinomial quadrático e cúbico para os solos (Figura 2). Nesse caso, o aumento na intensidade da moagem das amostras de malha de 2,000 $\mathrm{mm}$ para $1,700 \mathrm{~mm}$ ocasionou elevação nos teores de P extraído (Figura 2). Entretanto, quando essa moagem é intensificada até as malhas de 0,850 e $0,300 \mathrm{~mm}$, os valores de $\mathrm{P}$ extraído passam a diminuir novamente com valores semelhantes ao da peneira de malha de $2,000 \mathrm{~mm}$. Provavelmente, o aumento da superfície de contato pela intensificação da moagem até a malha de 1,700 $\mathrm{mm}$ expõe formas de $\mathrm{P}$ que não seriam acessadas pelo extrator; entretanto, a partir da malha de 0,850 $\mathrm{mm}$, esse $\mathrm{P}$ adicional extraído passa a ser readsorvido por grupos funcionais expostos pela moagem, equilibrando os valores de P extraído com o padrão de malha de $2,000 \mathrm{~mm}$ e, ou, a capacidade extrativa do método M3 passa a sofrer desgaste em razão do consumo dos íons $\mathrm{H}^{+}$pelos oxigênios expostos de grupos funcionais das partículas minerais. 


\section{Efeito do tipo de frasco}

Quando se utilizou frasco tipo snap-cap, o teor de P extraído pelo método M1 foi mais elevado em $73 \%$ dos solos do que os teores extraídos com uso de frasco tipo erlenmeyer (Quadro 3), independentemente da classe textural e do teor de $\mathrm{P}$ das amostras. Isso pode ser atribuído ao fato de a ação da solução extratora do M1 ser por dissolução ácida e no frasco tipo snap-cap ocorrer maior turbilhonamento no momento da agitação, bem como aumento na superfície de contato. Os extratos do M3 também apresentaram diferença significativa em $73 \%$ dos solos; entretanto, não houve comportamento uniforme quanto ao tipo de frasco e ao teor de $\mathrm{P}$ extraído. Nos solos LCm 1/A e LCl 1/A (teor de argila $>600 \mathrm{~g} \mathrm{dm}^{-3}$ e teor alto de P), o uso de frasco tipo snap-cap proporcionou maior extração de $\mathrm{P}$, da mesma forma o que ocorreu nos solos AIl 3/M e CBGm 3/M (teor de argila entre 210 e $400 \mathrm{~g} \mathrm{dm}^{-3} \mathrm{e}$ teor médio de P). Nos solos NFWc 1/B (teor de argila $>600 \mathrm{~g} \mathrm{dm}^{-3}$ ) e (teor baixo de P), PSMl 2/A, PSMl 3/A e PSMl 3/M, (teor de argila entre 410 e $600 \mathrm{~g} \mathrm{dm}^{-3} \mathrm{e}$ teor alto de P) e (teor de argila entre 210 e $400 \mathrm{~g} \mathrm{dm}^{-3} \mathrm{e}$ teor médio de $\mathrm{P}$ ), respectivamente, os extratos de $\mathrm{M} 3$ obtidos com o uso do frasco erlenmeyer apresentaram teores mais elevados de $\mathrm{P}$ extraído com relação aos obtidos com uso de frasco snap-cap.

Nos Estados do RS e de SC, os teores de P no solo são interpretados com base no teor de argila (CQFSRS/ SC, 2004), visto que esse fator altera a capacidade de extração do M1. Em trabalhos realizados com o extrator M3 não são mencionados efeitos do teor de argila sobre a capacidade de extração de $\mathrm{P}$ do solo (Gatiboni, 2003; Schlindwein, 2003; Gatiboni et al., 2005). Entretanto, resultados encontrados por Bortolon \& Gianello (2008), comparando os teores de P extraído pelos métodos M1 e M3, apontaram que em solos com teores de argila entre 410 e $600 \mathrm{~g} \mathrm{dm}^{-3} \mathrm{e}>600 \mathrm{~g} \mathrm{dm}^{-3}$ e teores menores que 20 e $10 \mathrm{mg} \mathrm{dm}^{-3} \mathrm{de} \mathrm{P}$, respectivamente, a solução M3 extraiu menos $\mathrm{P}$ que nas demais classes de argila.
Dessa forma, os resultados são semelhantes aos dos deste estudo, os quais evidenciaram que os teores de P extraído pelo M3 sofrem efeito do tipo de frasco, mas o teor de argila e os teores de $\mathrm{P}$ das amostras podem influir na extração de forma diferenciada.

\section{Efeito do volume vazio no frasco}

As regressões significativas entre os teores de $\mathrm{P}$ extraído pelas soluções de M1 e M3 e o volume vazio no frasco são apresentadas na figura 3. Nos extratos do extrator M1, houve efeito significativo $(p<0,05)$ em $100 \%$ das amostras para os teores de P extraído em razão do volume vazio no frasco, enquanto para M3 o efeito foi de $64 \%$ (Figura 3).

Os teores de P extraído pela solução utilizada no M1 diminuíram, consideravelmente, com a redução do volume vazio no frasco. Para os solos LFWc 1/B, LBVm 1/B, NFWc 2/MB, NFWm 2/MB e CBGm 3/M, houve comportamento linear no teor de P extraído com a diminuição do volume vazio, enquanto para os solos LCm 1/A, LCl 1/A, PSMl 2/A e PSMl 3/M houve comportamento polinomial quadrático e para os $\mathrm{PSMl}$ 3/A e AIl 3/M, cúbico, mas também, com tendência a diminuir (Figura 3). Ficou evidente que a ação do extrator M1 é potencializada quando se aumenta o volume vazio no frasco em razão, provavelmente, da maior intensidade de colisões entre as partículas e dessas com a parede do frasco, no momento da agitação da mistura solo:solução. Catani \& Nakamura (2012), estudando alterações na capacidade extrativa do método M1, observaram que, quando a quantidade de solo:solução variou de 5:100 para 20:100, a capacidade de extração de $\mathrm{P}$ da solução diminuiu, embora esse efeito possa ter sido provocado pela perda de força da solução extratora, por causa do volume utilizado e não, propriamente, do volume vazio no frasco. Em trabalho de Soares et al. (2012), os resultados diferiram dos encontrados, e o teor de P extraído aumentou com a diminuição do vazio do frasco. Entretanto, ressalta-se que nesses trabalhos a proporção da relação

Quadro 3. Efeito do uso de frasco de $50 \mathrm{~mL}$ tipo erlenmeyer e snap-cap sobre os teores de fósforo extraído pelos métodos Mehlich-1 e Mehlich-3

\begin{tabular}{|c|c|c|c|c|c|c|c|c|c|}
\hline \multirow{3}{*}{ Frasco } & \multicolumn{9}{|c|}{ Solo/Classe de argila } \\
\hline & $\mathrm{LCm}$ & LCl & LFWe & LBVm & PSMl & PSMl & AIl & CBGm & PSMl \\
\hline & $1 / \mathrm{A}$ & $1 / \mathrm{A}$ & $1 / \mathrm{B}$ & $1 / \mathrm{B}$ & $2 / \mathrm{A}$ & $3 / \mathrm{A}$ & $3 / \mathbf{M}$ & $3 / \mathbf{M}$ & $3 / \mathbf{M}$ \\
\hline & & & & & $\mathrm{P}\left(\mathrm{mg} \mathrm{L}^{-1}\right)$ & & & & \\
\hline & & & & & Mehlich-1 & & & & \\
\hline Erlenmeyer & 9,1 bA & $8,3 \mathrm{bB}$ & $2,2 \mathrm{~A}$ & $2,6 \mathrm{bA}$ & 9,8 bB & $20,4 \mathrm{bB}$ & $9,3 \mathrm{bB}$ & $11,1 \mathrm{bB}$ & $8,8 \mathrm{bB}$ \\
\hline Snap-cap & $12,2 \mathrm{aB}$ & $10,3 \mathrm{a}$ & $3,1 \mathrm{~A}$ & $4,1 \mathrm{aA}$ & $\begin{array}{c}11,2 \text { aA } \\
\text { Mehlich-3 }\end{array}$ & $30,9 \mathrm{aA}$ & $14,6 \mathrm{aA}$ & $16,0 \mathrm{aB}$ & $10,9 \mathrm{aA}$ \\
\hline Erlenmeyer & $10,2 \mathrm{bA}$ & $9,5 \mathrm{bA}$ & $2,2 \mathrm{aA}$ & $3,1 \mathrm{~A}$ & $13,3 \mathrm{aA}$ & $24,1 \mathrm{aA}$ & $12,7 \mathrm{bA}$ & $17,5 \mathrm{bA}$ & $12,4 \mathrm{aA}$ \\
\hline Snap-cap & $15,4 \mathrm{aA}$ & $11,2 \mathrm{a}$ & $1,7 \mathrm{bB}$ & $3,9 \mathrm{~A}$ & $9,4 \mathrm{bB}$ & $22,4 \mathrm{bB}$ & $15,5 \mathrm{aA}$ & $20,1 \mathrm{aA}$ & $10,8 \mathrm{bA}$ \\
\hline
\end{tabular}

Médias seguidas de letras minúsculas na coluna e dentro de cada extrator diferem entre si pelo teste de Tukey ( $<<0,05)$. Médias seguidas de letras maiúsculas na coluna, entre o mesmo frasco e entre extratores, diferem entre si pelo teste de Tukey $(p<0,05)$. 
solo:solução não foi mantida, logo, o efeito de variabilidade encontrado pode não ser em razão do vazio no frasco.

Quanto ao efeito do volume vazio, sobre os toeres de P extraído pela solução M3, houve tendência de comportamento polinomial quadrático e cúbico com relação aos solos (Figura 3). Entretanto, os teores de P extraído não seguiram a mesma tendência para todos os solos. Nos solos AIl 3/M, CGBm 3/M e LCm 1/A, os teores de $\mathrm{P}$ extraído foram mais elevadas com o aumento do volume vazio no frasco de 6,0 para $33,5 \mathrm{~mL}$; no entanto, quando o volume vazio aumentou de 33,5 para $39,0 \mathrm{~mL}$, os teores de $\mathrm{P}$ extraído diminuíram. Para o solo NFWm $2 / \mathrm{MB}$, os teores de $\mathrm{P}$ extraído não diferiram para o volume vazio entre 6,0 e 33,5 mL, mas quando o volume passou a ser de 39,0 $\mathrm{mL}$ o teor de P extraído aumentou. Paro o solo LCl 1/A, os teores de $\mathrm{P}$ extraído elevaram-se com o aumento do volume vazio no frasco de 6,0 para $22,5 \mathrm{~mL}$ e passaram a diminuir quando o volume vazio foi de 33,5 até 39,0 mL (Figura 3). Nos solos PSMl 2/A e PSMl 3/A, os teores de $\mathrm{P}$ extraído diminuíram quando volume vazio passou de 6,0 para $17,0 \mathrm{~mL}$, aumentando esse teor quando foi de 17,0 para $22,5 \mathrm{~mL}$ e reduzindo novamente quando o volume vazio foi de 33,3 até 39,0 mL.

Para o solo PSMl 3/M, os teores de P extraído se elevaram quando o volume vazio aumentou de 6,0 até $22,5 \mathrm{~mL}$ e, novamente, decresceram quando o volume vazio no frasco aumentou de 33,5 até 39,0 mL.

Dessa forma, observou-se que, para o extrator M3, os teores de $\mathrm{P}$ extraído não foram influenciadas somente pelo contato e pela cinética de colisões no momento da agitação, como ocorreu para o M1. Para esse extrator, além do efeito do volume vazio no frasco, pode haver um efeito resultante da interação do teor de $\mathrm{P}$ e teor e tipo de argila da amostra de solo. Assim, os teores de $\mathrm{P}$ extraído pelo método M3 não seguiram a mesma tendência em relação aos teores observados no método M1 (Figura 3). Isso pode estar relacionado à reação de extração do extrator M3 ser diferenciada do extrator M1, em que a reação ocorre por dissolução ácida dos coloides inorgânicos, o que a torna dependente do contato dos íons $\mathrm{H}^{+}$com o fosfato ligado à fase sólida do solo. No caso do extrator M3, além da ação ácida, ocorre uma reação de troca de ligantes do $\mathrm{P}$ ligado, principalmente ao $\mathrm{Al}$ e Fe com o flúor ( $\left.\mathrm{F}^{-}\right)$, reação essa menos dependente das colisões; sendo assim, é possível que essa seja menos influenciada pelo volume vazio no frasco.

\section{CONCLUSÕES}

1. Quando for utilizado o método M3 para extração do $\mathrm{P}$, deve-se padronizar a intensidade da moagem das amostras de solo. Isso não é necessário para o M1.

2. O frasco tipo snap-cap superestima a quantidade de $\mathrm{P}$ extraído pelo método $\mathrm{M} 1$ em relação ao tipo erlenmeyer, sendo necessário um único modelo para ser utilizado em laboratórios de análise de solo para extração do P.

3. A relação volume da amostra $\left(\mathrm{cm}^{3}\right)$ para volume de solução extratora $\left(\mathrm{cm}^{3}\right)$ não deve sofrer alteração na sua quantidade, devendo ser mantida em 3:30, para evitar o efeito do volume vazio no frasco na quantidade de P extraído pelos métodos M1 e M3.

\section{AGRADECIMENTOS}

À FAPERGS, ao CNPq e à CAPES, pelo auxílio financeiro e pelas bolsas de Mestrado, Doutorado e Produtividade em Pesquisa dos autores.

\section{LITERATURA CITADA}

BAHIA FILHO, A.F.C.; BRAGA, J.M.; RIBEIRO, A.C. \& NOVAIS, R.F. Sensibilidade de extratores químicos à capacidade tampão de fósforo. R. Bras. Ci. Solo, 7:243249, 1983.

BORTOLON, L. \& GIANELLO, C. Fósforo extraído pela solução de Mehlich-1 determinado por colorimetria e ICP em solos do sul do Brasil. R. Bras. Ci. Solo, 34:263-268, 2010 .

BORTOLON, L. \& GIANELLO, C. Interpretação de resultados analíticos de fósforo pelos extratores Mehlich-1 e Mehlich3 em solos do Rio Grande do Sul. R. Bras. Ci. Solo, 32:2751$2756,2008$.

BORTOLON, L. Métodos de avaliação da disponibilidade de nutrientes para as plantas em solos do Rio Grande do Sul. Porto Alegre, Universidade Federal do Rio Grande do Sul, 2005. 129p. (Dissertação de Mestrado)

BRASIL, E.C. \& MURAOKA, T. Extratores de fósforo em solos da Amazônia tratados com fertilizantes fosfatados. R. Bras. Ci. Solo, 21:599-606, 1997.

CAJUSTE, L.J. \& KUSSOW, W.R. Use and limitations of the North Carolina method to predict available phosphorus in some Oxisols. Trop. Agric., 51:246-252, 1974.

CATANI, R.A. \& NAKAMURA, P.N. Extração do fósforo nativo e do adicionado ao solo com várias soluções. Disponível em: $<$ http://www.scielo.br/scielo.php?script=sci_arttexr\& pid=S0071-12761971000100021\&Ing=en\&nrm=iso>. Acesso em: 20 dez. 2012.

COMISSÃO DE QUÍMICA E FERTILIDADE DO SOLO . CQFSRS/SC. Manual de recomendação de adubação e de calagem para os Estados do Rio Grande do Sul e Santa Catarina. 10.ed. Porto Alegre, SBCS/NRS, 2004. 400p.

FERREIRA, D.F. SISVAR: Um programa para análises e ensino de estatística. R. Symposium, 6:36-41, 2008. 
GATIBONI, L.C. Disponibilidade de formas de fósforo do solo às plantas. Santa Maria, Universidade Federal de Santa Maria, 2003. 247p. (Tese de Doutorado)

GATIBONI, L.C.; KAMINSKI, J. \& RHEINHEIMER, D.S. Modificações nas formas de fósforo do solo após extrações sucessivas com Mehlich-1, Mehlich-3 e resina trocadora de ânions. R. Bras. Ci. Solo, 29:363-371, 2005.

MAKAROV, M.I.; MALYSHEVA, T.I.; HAUMAIER, L.; ALT, H.G. \& ZECH, W. Organic phosphorus compounds in particle-size fractions of mountain soils in the northwestern Caucasus. Geoderma, 118:101-114, 2004.

MEHLICH, A. Determination of $\mathrm{P}, \mathrm{Ca}, \mathrm{Mg}, \mathrm{K}, \mathrm{Na}$ and $\mathrm{NH}_{4}$ by North Carolina soil testing laboratories. Raleigh, University of North Carolina, 1953.

MEHRA, O.P. \& JACKSON, M.L. Iron oxide removal from soils by a dithionite-citrate system buffered with sodium bicarbonate. Clays Clays Miner., 7:317-327, 1964.

PROGRAMA DE CONTROLE DE QUALIDADE DA ROLAS ROLAS-RS/SC. Disponível em: <http:// www.cnpt.embrapa.br/pesquisa/solos/rolas.htm $>$. Acesso em: 10 maio 2012.

RAIJ, B.van; QUAGGIO, J.A.; CANTARELLA, H.; FERREIRA, M.E.; LOPES, A.S. \& BATAGLIA, O. Análise química do solo para fins de fertilidade. Campinas, Fundação Cargill, 1987. 170p.

RHEINHEIMER, D.S.; GATIBONI, L.C. \& KAMINSKI, J. Fatores que afetam a disponibilidade do fósforo e o manejo da adubação fosfatada em solos sob sistema plantio direto. Ci. Rural, 38:576-586, 2008.
ROLIM, M.V.; NOVAIS, R.F.; NUNES, F.N. \& ALVARES V., V.H. Efeito da moagem do solo no teor de fósforo disponível por Mehlich-1, resina em esferas e em lâminas. R. Bras. Ci. Solo, 32:1181-1190, 2008.

SCHLINDWEIN, J.A. Calibração de métodos de determinação e estimativa de doses de fósforo e potássio em solos sob plantio direto. Porto Alegre, Universidade Federal do Rio Grande do Sul, 2003. 186p. (Tese de Doutorado)

SIKORA, F.J.; HOWE, P.S.; HILL, L.E.; REID, D.C. \& HAROVER, D.E. Comparison of Colorimetric and ICP determination of phosphorus in Mehlich 3 soil extracts. Commun. Soil Sci. Plant Anal., 36:875-887, 2005.

SILVA, F.C. \& RAIJ, B.van. Disponibilidade de fósforo em solos avaliada por diferentes extratores. Pesq. Agropec. Bras., 34:267-288, 1999

SOARES, R.; ESCALEIRA, V.; CAMPOS, D.V.B.; MONTEIRO, M.I.C.; CARNEIRO, M.C.; SANTELLI, R.E. \& BERNARDI, A.C.C. Comparação de métodos convencional e alternativo para determinação de sódio, potássio e fósforo em extratos de solos obtidos com solução de Mehlich-1. R. Bras. Ci. Solo, 36:105-112, 2012.

TEDESCO, M.J.; GIANELLO, C.; BISSANI, C.A.; BOHNEN, H. \& VOLKWEISS, S.J. Análise de solo, plantas e outros materiais. 2.ed. Porto Alegre, Universidade Federal do Rio Grande do Sul, 1995. 174p.

VOLLHARDT, K.P.C. \& SCHORE, N.E. Química orgânica: Estrutura e função. 4.ed. Porto Alegre, Bookman, 2004. 1112p.

WANG, S.; JIN, X.; BU, Q.; ZHOU, X. \& WU, F. Effects of particle size, organic matter and ionic strength on the phosphate sorption in different trophic lake sediments. J. Hazard. Mater., 128:95-105, 2006. 